\title{
Anger and aggression in the science
}

Ulysses Paulino Albuquerque ${ }^{1}$ and Rômulo Romeu Nóbrega Alves ${ }^{2 *}$

\author{
1 Laboratório de Ecologia e Evolução de Sistemas Socioecológicos, Departamento de Botânica, Universidade Federal de \\ Pernambuco, 50670901 Recife, PE, Brazil \\ 2 Departamento de Biologia, Universidade Estadual da Paraíba, Av. Baraúnas \#351, Bodocongó, Campina Grande, PB, 58430 \\ 335, Brazil \\ * Corresponding author.. Email address:UPA (upa677@hotmail.com), RRNA (romulo_nobrega@yahoo.com.br)
}

A few years ago an article was written demonstrating concern about one of the essential aspects of scientific communication: peer review (Albuquerque 2011). The text addressed the issue of the scarcity of good reviewers, and their burden of work, especially considering the increasing number of scientific journals created in recent decades. At the time, the question was whether we could be facing a "tragedy" due to the lack of reviewers. In our view, there is an associated problem posed by Virginia Walbot (2009): Are we training pit bulls to review our manuscripts? Anger and aggressive editors and reviewers are a growing problem in science and it is characterized by a type of unethical behavior. Lately there has been a fertile discussion about misconduct and ethics in science. From the early identification of misconduct in science to the way leading investigators deal with their work teams.

The peer review system is considered to be at the heart of modern science; it is a tool used since the creation of the first scientific journals and it contributes to the selection and quality of published articles. Despite its importance, and the credibility it generates in the system, it is not free of bias. There is extensive literature on this subject, but relatively little has been researched, to the best of our knowledge, on the aspects pointed out in the previous paragraph. The experience of submitting an article to a journal is much more than just following a few simple steps outlined in the journal guidelines. In general, a scientific article is a piece of science that requires much effort, time and investment by its authors. In our view, the experience of submitting the article to the peer review process needs to be gratifying, representing an important step in the training of researchers. When we say gratification, we do not mean that it comes solely from the acceptance of the manuscript. The rejection of a paper can be a very rich experience for authors, especially when they receive mature, objective and clear feedback about the research. Of course, rejection does not translate into happiness, but after the initial shock, we authors can take advantage of good criticism.

However, this experience can be extremely negative, especially for young researchers, when they receive reviews that suggest that the reviewer has not read the material, or that he or she has some conflict of interest with respect to the authors' research, or when the reviewer's criticism is very superficial. Even worse are the ad hominem critiques that focus not on the quality of the material but on the authors. There is no justification for supporting these 
personal, cowardly and unprofessional attacks (especially since the reviewers are protected by anonymity). Such behavior should be vigorously discouraged by editors in two ways: 1. Not considering aggressive reviews for article evaluation; 2. Removing from their review board researchers who systematically behave in such an unprofessional manner. When this stance comes from associate editors, authors should be encouraged to complain to the editorinchief or the journal publishers.

There is no doubt that there are several academic styles of review, and that some reviews are much better than others (see Ward 2016), but it is our duty as editors to think about the "health" of the peer review system by curbing behaviors that harm the system and the researchers (in terms of a productive experience). A recent article (Silbiger and Stubler 2019) found that such behavior is common and it affects groups traditionally underrepresented in science. Some examples of unprofessional reviews may make our point clearer (from Silbiger and Stubler 2019):

- "Despite being a woman, the PI was trained by several leading men in the field and is thus likely adequately prepared to lead the proposed research";

- "What the authors have done is an insult to science";

- "You should look closely at a career outside of science";

- "This is obviously written by a group from a lower standardized institution based on the quality of work".

Silbiger and Stubler (2019) define in their study unprofessional and unethical behavior as "(1) lack constructive criticism, (2) are directed at the author(s) rather than the nature or quality of the work, (3) use personal opinions of the author(s)/work rather than evidencebased criticism, or (4) are "meanspirited" or cruel". And why is this behavior not restricted? Because often authors (especially young researchers) are afraid to write to editors to point out the problem. We must introduce the culture of writing to editors-with the respect and education we would like to have received-whenever, as authors, we have fallen victim to angry reviewers / editors. Similarly, both publishers and scientific societies must develop codes of ethics and good practice in peer review to restrain such practices (see Silbiger and Stubler 2019), while at the same time policing the system to identify nonprofessional reviewers. We believe that only in this way will the system be capable of identifying researchers who seem to have no concern for the development of a quality, balanced, fair and respectful science.

We conclude with Silbiger and Stubler (2019):

"(...) that unprofessional peer reviews are pervasive and that they disproportionately harm underrepresented groups in STEM. Specifically, underrepresented groups were most likely to report direct negative impacts on their scientific aptitude, productivity and career advancement after receiving an unprofessional peer review. While it was beyond the scope of this study, future investigations should also focus on the effect of unprofessional peer reviews on first generation scientists English as a second language, career stage, peer review in grants, and other factors that could lead to differences in downstream effects. Unprofessional peer reviews have no place in the scientific process and individual 
scientists have the power and responsibility to enact immediate change. However, we recognize and applaud those reviewers and editors (and there are many!) that spend a significant amount of time and effort writing thoughtful, constructive, and detailed criticisms that are integral to moving science forward".

\section{REFERENCES}

Albuquerque UP (2011) The tragedy of the common reviewers the peer review process. Revista Brasileira de Farmacognosia 21:13. Doi: 10.1590/S0102695X2011005000036

Silbiger NJ, Stubler AD (2019) Unprofessional peer reviews disproportionately harm underrepresented groups in STEM. PeerJ 7: e8247 https://doi.org/10.7717/peerj.8247

Walbot $V(2009)$ Are we training pit bulls to review our manuscripts?. Journal of Biology 8 , 24. Doi:10.1186/jbiol125

Ward K (2016) Towards a typology of academic peer review styles. Urban Geography 37: 651654 Doi: 10.1080/02723638.2016.1190559 